\title{
Enhancing visual preference of ecological rehabilitation sites
}

\author{
Denise E. Hands, Robert D. Brown \\ Article History: \\ Received 4 October 2000 \\ Received in revised form March 92001 \\ Accepted 29 October 2001
}

\begin{abstract}
NOTICE: This is the authors' version of a work that was accepted for publication in LANDSCAPE AND URBAN PLANNING: AN INTERNATIONAL JOURNAL OF LANDSCAPE ECOLOGY, PLANNING AND DESIGN. Changes resulting from the publishing process, such as peer review, editing, corrections, structural formatting, and other quality control mechanisms, may not be reflected in this document. Changes may have been made to this work since it was submitted for publication. A definitive version was subsequently published in LANDSCAPE AND URBAN PLANNING: AN INTERNATIONAL JOURNAL OF LANDSCAPE ECOLOGY, PLANNING AND DESIGN, VOLUME 58(2002) 57-70. If you refer to this work please cite the published version.
\end{abstract}




\begin{abstract}
A study was undertaken to assess visual preference of ecological rehabilitation of decommissioned industrial lands. Computerized visual simulations were used to illustrate the effects of alternative design treatments at two stages of development: establishment and maturity. Employees who work at the industrial site were used as a subject group and were asked to complete a questionnaire, with a resulting response rate of $65 \%(n=60)$. Results indicated that the common perception that rehabilitation sites are 'messy' was also a concern in this industrial site. However, increasing the amount and diversity of color, as well as visible indicators of 'human intent' for the landscape, increased preference levels, as indicated by rank coefficient analysis and multi-dimensional scaling (MDS). There was no statistically significant difference between preference levels at establishment and at maturity of the landscape. Design implications arising from the results of this study included: (a) a significant increase in visual preference was achieved through the use of 'vernacular cues to care' such as the addition of cultural elements like bird boxes and large rocks; (b) clustering and banding of vegetation had no apparent effect on visual preference; (c) sparseness of vegetation was a major reason for negative preference, thus any technique that contributes to the rapid formation of ground cover should increase visual preference; and (d) the amount and diversity of color had a substantial effect on visual preference and should be maximized.
\end{abstract}




\section{Introduction}

Much of the surface of the earth has been modified through anthropomorphic development. As the economic value of a landscape diminishes it often becomes abandoned. These abandoned landscapes, if left with- out intervention, will eventually rehabilitate naturally through ecological processes. However, these processes can take a long time, and the interim landscapes have been shown to be of low scenic preference, especially throughout the early successional stages.

Abandoned landscapes have the potential to improve the biodiversity and ecological function of an area, as well as provide accessible `natural' areas for public use. One of the biggest problems with rehabilitation projects has been public perception. It seems that although people have a visual preference for natural over built areas, what people perceive as 'natural' is often quite different in appearance from naturalized areas that are high in ecological function. In fact, the general public often disapproves of the appearance of areas that are high in ecological function, describing them as messy and unkempt (e.g. Barro and Bright, 1998; Gobster, 1994; Nassauer, 1988, 1995, 1997; Schulhof, 1989). This negative perception is especially prevalent during the establishment phases, which can be quite extended (Maag, 1995; Raffetto, 1993).

As rehabilitation/naturalization projects are often in highly visible and accessible areas, the support of the general public is essential for the long-term success of these projects and the ecological benefits that they provide $\square$ Nassauer, 1997). Interpretive signage and education programs have been shown to improve preference; however, low visual preference for naturalized areas is still a chronic problem (Barro and Bright, 1998; Hough, 1989; Keyes, 1984; Maag, 1995;Schulhof,1989). Little research has been devoted to examining how design may be incorporated into such projects to improve their aesthetic appeal especially during the critical establishment stages. This study will begin to examine the ameliorating potential of specific design elements on visual preferences for rehabilitating landscapes.

Some studies have examined the public's preferences in regards to ecologically functioning landscapes in urban, suburban, and rural landscapes, but there has been little work that assesses preferences in the context of functioning industrial landscapes. Research has also shown that employees can have different visual preferences than residents for the same landscapes $\square$ Gallager, 1977; Kaplan,1993).This study investigated the visual preferences of employees working at the Cytec Canada Welland Plant in Niagara Falls, Ont., Canada for an ongoing rehabilitation project that is being undertaken on the property of their corporate headquarters.

\section{Study site}

The corporate headquarters of Cytec occupy an 800-acre property adjacent to the Welland River in the city of Niagara Falls, Canada (see Fig.1). The facility was constructed during World War II as an ammunition factory, and was converted to 
fertilizer production in the post-war years. Currently, phosphine products are manufactured on site. The operating plant occupies only a small portion of the total land, and employs approximately 100 people. Much of the land has remnants of industrial production, including surface deposits of cinder and gravel, soil compaction, leveled buildings with backfield basements, and varying levels of nitrate, ammonia, and phosphate contamination of the soil.

Cytec's primary motivation for initiating rehabilitation was to improve the aesthetic quality of this area for employees, neighbors, and clients. Approximately

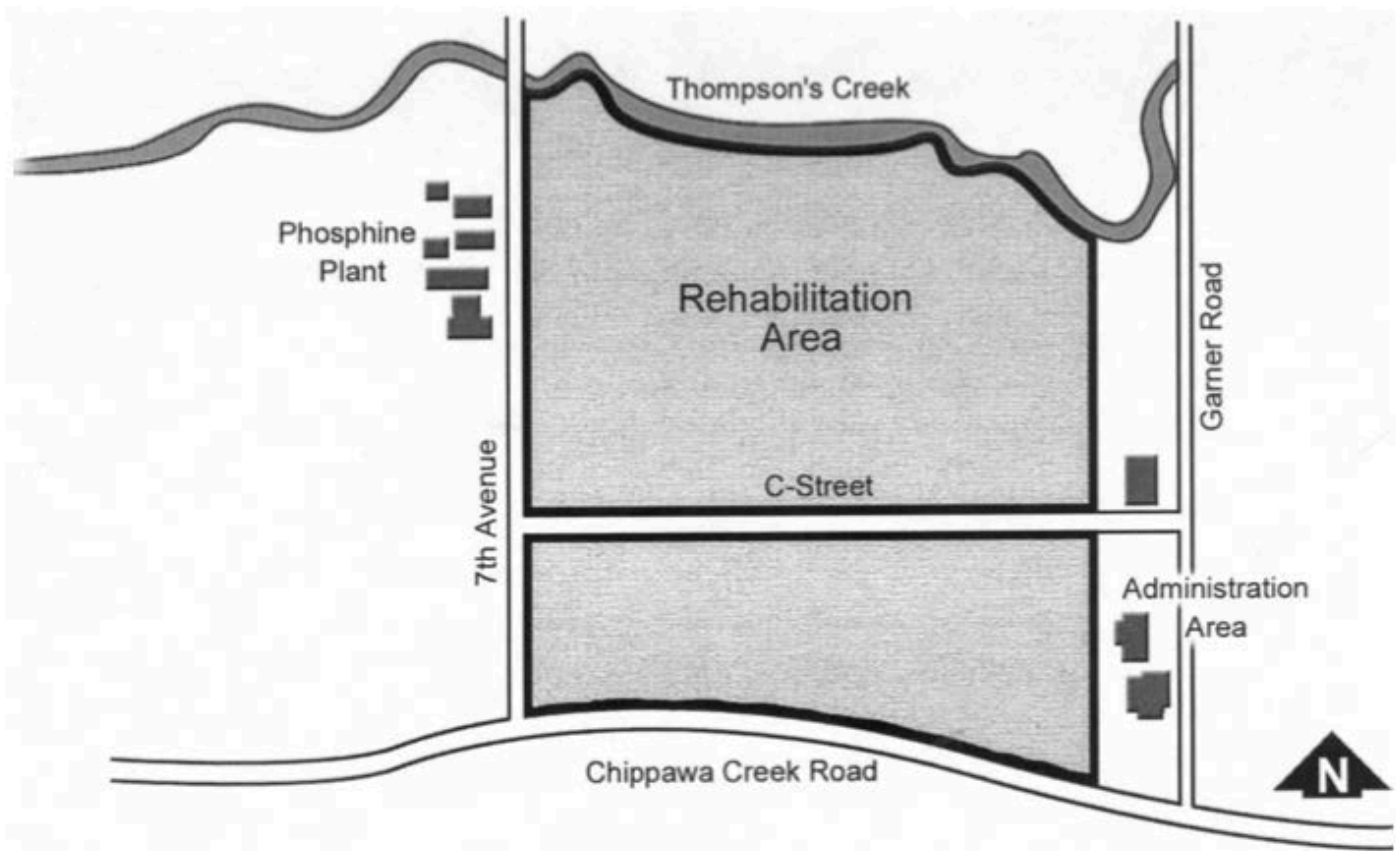

Fig. 1. Site map of Cytec Canada rehabilitation project.

200 acres in the central area of the site have recently undergone active demolition, and are the main focus of the rehabilitation work. This area is traversed on a daily basis by employees on their way to work or moving between the administration area and the production area, and thus is of high visual priority.

Several rehabilitation and management strategies are currently being employed on the property, including natural regeneration (essentially a 'hands-off' approach), managed succession (a non-planting, ‘accelerated succession' approach), and plantation establishment and management of the desired plant species). The plantation approach has the advantage of allowing more design intervention, and has a more predictable resultant landscape. It is the plantation areas of the rehabilitation project that were used in this study. 


\section{Method}

This study utilized computerized visual simulations of landscapes at establishment and mature stages of development, and through a questionnaire asked employees to indicate their visual preference of various different landscape development approaches.

\subsection{Photographic representation}

To ensure that subjects were responding to research variables, each simulation in a set used the same base landscape view. Every attempt was made to ensure the chosen view was representative of the site as a whole. This task was facilitated by the fact that the rehabilitation site was fairly homogeneous, consisting of early successional meadow species with little topographic change.

The base photo was selected to represent a typical scene in the rehabilitation area which contained elements such as a building, fencing, and a decommissioned are hydrant to provide an identifiable context. It was also selected to be adjacent to the Cytec plant entrance so that it was passed daily by all subjects while entering the site, and should have been equally familiar to all. The viewing distance for the simulations was $30 \pm 45 \mathrm{ft}$ so as to approximate the distance and scale at which the landscape would typically be viewed from a car traveling along the main route through the plant. The base photo was overlain with various computer images to provide a range of landscape treatments for the same site, holding as many variables constant as possible. Source images used to create the manipulated images included: site photos taken during the first year of implementation of the rehabilitation project, photos of similar rehabilitation projects through- out Ontario, photos of natural communities throughout Ontario, and native plant nursery catalogue photos.

All source images were digitally scanned using a Hewlett Packard (4C/T) flatbed

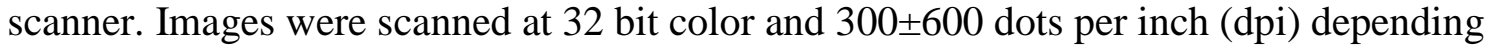
on the source media (i.e. photos and print media were scanned at $300 \mathrm{dpi}$, while slides were scanned at $600 \mathrm{dpi}$ ). These levels were more than sufficient for the purposes of preference rating (e.g. Orland, 1994; Ye, 1993). Once scanned, the photos were manipulated using a Pentium 166 computer with Adobe PhotoShop 5.0 software. The manipulated photos were eventually printed at 720 dpi x 720 dpi using a Cannon BJ 5000 seriescolor inkjetprinter. Two 4 in x 6 in. photos were displayed per $8 X 5$ in $x 11$ in. sheet of paper.

The major dependent variable was visual preference for the rehabilitating landscapes. Two main independent variables, 'color' and `apparent human intent', were evaluated at both establishment and mature landscape stages. A limited range of landscape treatment variables were used to ensure that each variable could be examined in more depth. Within the variable 'color' both total amount of color, and diversity of colors in the image were considered. As green is the pervasive landscape hue in mature grassland communities, for the purposes of this study it was considered as background 
and was not counted as a distinct hue. Similarly, a brown matrix representing wood chip mulch) was used as background for the early stages of planting, and was not considered a hue for analysis purposes. These background hues were kept constant throughout the simulation.

Design elements such as clustering and banding of color/texture, as well as inclusion of non-vegetative evidence of human intervention in the landscape, such as rocks, a constructed bird box, and signage were considered for the variable `apparent human intent'.

Seven color manipulations and nine apparent human intent manipulations were created for each of the two stages resulting in a total of 32 images. The use of simulation allowed scenes to be compared with only a

Table 1

Computerized visual simulations for study

\begin{tabular}{|c|c|c|}
\hline \multirow[t]{2}{*}{ Stage } & \multicolumn{2}{|l|}{ Description of manipulation } \\
\hline & Variable: color & Variable: apparent human intent \\
\hline Establishment & No color (except green) & Existing site conditions \\
\hline Maturity & No color (except green) & Natural regeneration \\
\hline Establishment & $\begin{array}{l}\text { Low color }(\sim 30 \%) \\
\text { Low complexity/diversity (one hue) }\end{array}$ & Natural scatter pattern \\
\hline Maturity & $\begin{array}{l}\text { Low color }(\sim 30 \%) \\
\text { Low complexity/diversity (one hue) }\end{array}$ & Natural grassland \\
\hline Establishment & $\begin{array}{l}\text { Low color }(\sim 30 \%) \\
\text { Medium complexity/diversity (three hues) }\end{array}$ & Small clusters (of a single species) \\
\hline Maturity & $\begin{array}{l}\text { Low color }(\sim 30 \%) \\
\text { Medium complexity/diversity (three hues) }\end{array}$ & Natural scatter pattern (no clusters) \\
\hline Establishment & $\begin{array}{l}\text { Low color }(\sim 30 \%) \\
\text { High complexity/diversity (over five hues) }\end{array}$ & Large clusters (of a single species) \\
\hline Maturity & $\begin{array}{l}\text { Low color }(\sim 30 \%) \\
\text { High complexity/diversity (over five hues) }\end{array}$ & Small clusters (of a single species) \\
\hline Establishment & $\begin{array}{l}\text { High color }(\sim 70 \%) \\
\text { Low complexity/diversity (one hue) }\end{array}$ & Linear bands (of a single species) \\
\hline Maturity & $\begin{array}{l}\text { High color }(\sim 70 \%) \\
\text { Low complexity/diversity (one hue) }\end{array}$ & Large clusters (of a single species) \\
\hline Establishment & $\begin{array}{l}\text { High color }(\sim 70 \%) \\
\text { Medium complexity/diversity (three hues) }\end{array}$ & Linear bands (with horticultural labeling stakes) \\
\hline Maturity & $\begin{array}{l}\text { High color }(\sim 70 \%) \\
\text { Medium complexity/diversity (three hues) }\end{array}$ & Linear bands \\
\hline Establishment & $\begin{array}{l}\text { High color }(\sim 70 \%) \\
\text { High complexity/diversity (over five hues) }\end{array}$ & Addition of constructed birdbox (no other intervention) \\
\hline Maturity & $\begin{array}{l}\text { High color }(\sim 70 \%) \\
\text { High complexity/diversity (over five hues) }\end{array}$ & Constructed birdbox (added to natural regeneration image) \\
\hline Establishment & & Addition of large rocks (arranged to add visual structure to the vegetation) \\
\hline Maturity & & Addition of large rocks (arranged to add visual structure to the vegetation) \\
\hline Establishment & & Addition of birdbox and interpretive signage (no other intervention) \\
\hline Maturity & & Addition of birdbox and interpretive signage (no other intervention) \\
\hline
\end{tabular}


single variable altered. Table 1 outlines how the independent variables were manipulated across the set of photographs. A sample of the photographs, showing the range of treatments, is illustrated in Fig. 2.

\subsection{Questionnaire}

The simulated photos were divided into four sets, representing an early and mature stage for each of the two independent variables. The photos were placed in a random order within each set, and this order was kept constant for all subjects. Subjects were asked to rate each photo on a 7-point likert-type scale based on how much they visually preferred the scene within the context of their site. On the scale, a rating of ' 1 ' indicated that they strongly disliked a scene, and a '7' indicated that they strongly liked a scene. Subjects were instructed to review all the photos in a given set before individually rating them. Respondents were also encouraged to use the full range of the scale. At the end of each set, subjects were asked to describe in one or two words, what they liked about their highest rated photo, and what they disliked about their lowest rated photo.

Upon completion of the questions on preference, subjects were asked to fill in a brief questionnaire about socio-demographic information and about their attitudes towards rehabilitation. Socio-demographic characteristics polled included: gender, age range, job title, education level, length of time working for Cytec, membership in environmentally-oriented associations, and level of participation in outdoor activities. Several open-ended questions at the end of the survey allowed respondents to make additional comments concerning their aesthetic vision for the site and/or problems that they foresaw.

\subsection{Subject group}

There is no public access to the site, so the only subject group available for study was employees of Cytec Canada. A comparison between responses of employees and people unfamiliar with the site would provide for interesting future research, but such a comparison was beyond the scope of this study.

\subsection{Data collection}

The questionnaire was pretested, then distributed to Cytec Canada employees on 29 April 1999 via intra-company mail. Cytec had 101 official employees at the Welland Plant, however, for various reasons, only 93 were on standard payroll on the study date. Due to the small population size, we decided to survey all employees. The surveys were attached to employees' regular payroll checks to ensure that all would receive them. This distribution method was chosen to cause minimal amount of disturbance to Cytec's normal company activity. As well, a number of employees worked in restricted access areas where it was not possible to survey them in a more direct manner.

Ten sets of photographic simulations were produced and bound and these booklets were placed on depart- mental bulletin boards in lunch rooms and central meeting areas. 


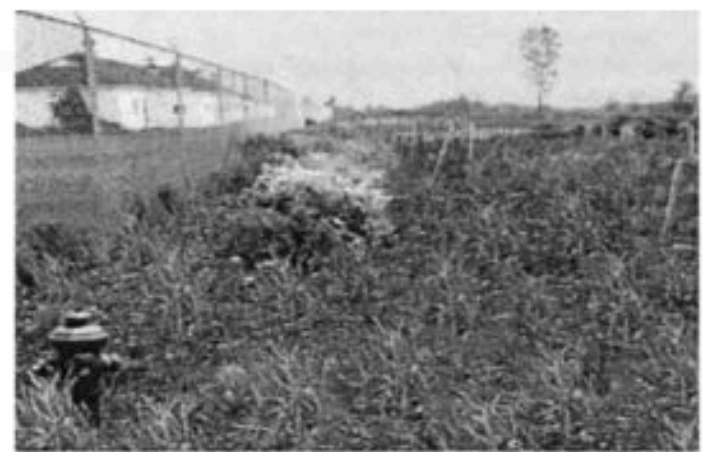

Establishment: No Colour

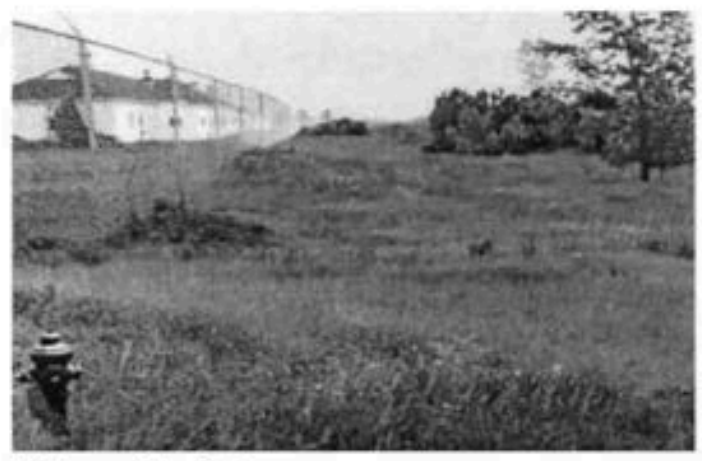

Mature: No Colour

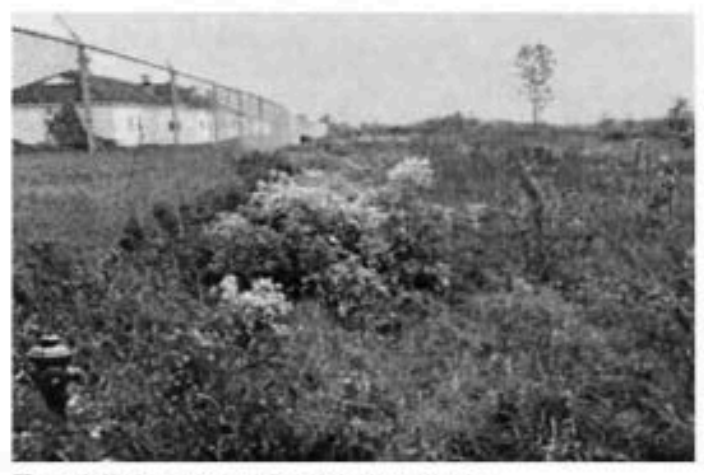

Establishment: No Intervention

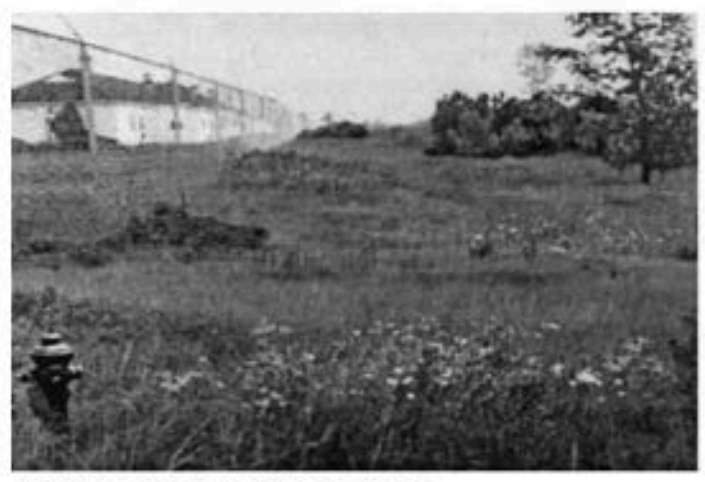

Mature: Natural Grassland

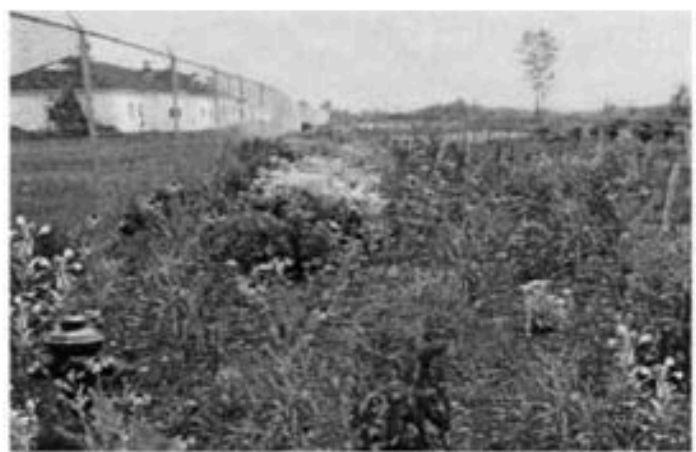

Establishment: High Colour

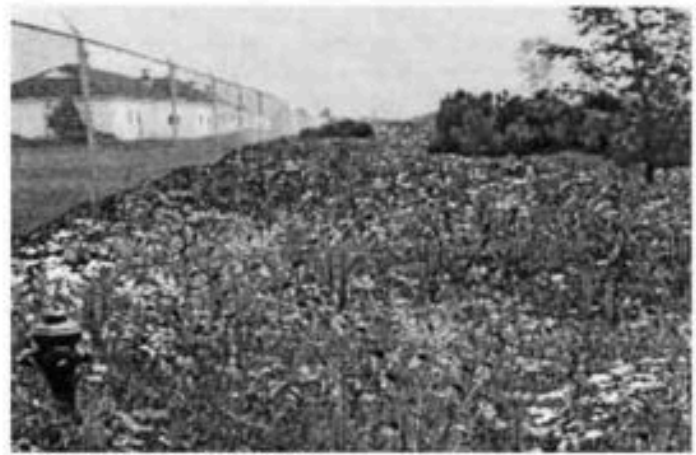

Mature: High Colour

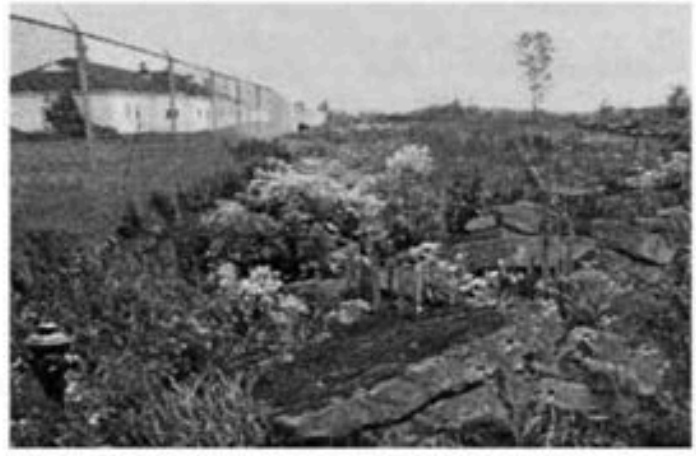

Establishment: Addition of Rocks

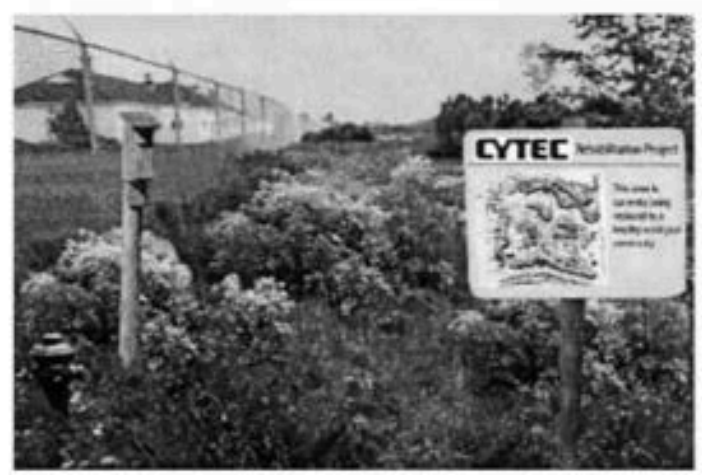

Mature: Natural Regeneration with Signage and Birdbox

Fig. 2. Sample of visual simulations used in the study. 
A cover letter attached to the questionnaires instructed employees where to find the photographs, as well as described the purpose of the study. Employees filled in the surveys on their own time on coffee and lunch breaks. A follow-up session was held 2 weeks later at a company barbeque. Although subjects could not be supervised while they completed the preference study (due to the restricted nature of their work area) the size of the simulated images, and the fact that they were bound together in booklet form, discouraged multiple subjects from viewing the simulations at the same time.

Observations during the follow-up session indicated that most users completed the study in $10-12$ min.

Data collected were analyzed through the use of the SPSS statistical analysis package version 8.0 and Microsoft Excel '97. Subject demographic information was described using descriptive statistics. Visual preferences for each photograph (being ordinal data) were described by determining the mode, median, range, and inter-quartile range of the total preference rating. In addition, the frequency of each rating given by respondents was calculated for each simulation.

Several statistical analyses were conducted on the data. Spearman's rank coefficient was used to assess correlation's between independent and dependent variables. Since Spearman's coefficient also provides a numerical description of the strength of a correlation, it was possible to evaluate the relative importance of each independent variable. It was also possible to assess potential changes in the relative importance of a particular variable over time by comparing the Spear- man's coefficient for the two stages of the same independent variable.

A multi-dimensional scaling (MDS) analysis was conducted to group photographs according to their similarities in their preference ratings. The Kolmogorov-Smirnov test, the Kruskal-Wallis test, and the median test were used to evaluate intergroup differences. Finally, qualitative content analysis was per- formed on all text-based responses.

\section{Results and analysis}

Fourty-two surveys were completed after the initial distribution, and a further 18 were collected at the follow-up session, resulting in a total of sixty completed surveys and a response rate of approximately65\%. The Kolmogorov-Smirnov test, the KruskalWallis test, and the median test did not reveal any significant in influence of demographic characteristics on employee visual preferences. 
Table 2

Measures of central tendency for independent variable color

\begin{tabular}{|c|c|c|c|c|c|c|c|c|}
\hline \multirow[t]{2}{*}{$\begin{array}{l}\text { Photo } \\
\text { number }\end{array}$} & \multicolumn{2}{|c|}{ Mode } & \multicolumn{2}{|c|}{ Median } & \multicolumn{2}{|c|}{ Range } & \multicolumn{2}{|c|}{$\begin{array}{l}\text { Inter-quartile } \\
\text { range }\end{array}$} \\
\hline & $E^{a}$ & $\mathrm{M}^{\mathrm{b}}$ & E & $\mathrm{M}$ & E & M & E & M \\
\hline 1 & 1 & 1 & 3 & 2 & 6 & 6 & 3 & 3 \\
\hline 2 & 5 & 4 & 5 & 4 & 6 & 6 & 3 & 2 \\
\hline 3 & 4 & 4 & 4 & 5 & 6 & 7 & 1 & 2 \\
\hline 4 & 5 & 4 & 5 & 5 & 5 & 7 & 2 & 2 \\
\hline 5 & 4 & 3 & 4 & 4 & 6 & 6 & 1.5 & 2 \\
\hline 6 & 6 & 6 & 6 & 5 & 6 & 6 & 2 & 2 \\
\hline 7 & 7 & 7 & 7 & 5 & 6 & 7 & 3 & 3 \\
\hline
\end{tabular}

${ }^{a}$ E: establishment stage.

${ }^{\mathrm{b}}$ M: mature stage.
Table 3

Measures of central tendency for independent variable apparent human intent

\begin{tabular}{|c|c|c|c|c|c|c|c|c|}
\hline \multirow[t]{2}{*}{$\begin{array}{l}\text { Photo } \\
\text { number }\end{array}$} & \multicolumn{2}{|c|}{ Mode } & \multicolumn{2}{|c|}{ Median } & \multicolumn{2}{|c|}{ Range } & \multicolumn{2}{|c|}{$\begin{array}{l}\text { Inter-quartile } \\
\text { range }\end{array}$} \\
\hline & $\mathrm{E}^{\mathrm{a}}$ & $\mathrm{M}^{\mathrm{b}}$ & $\mathrm{E}$ & M & E & M & $\mathrm{E}$ & $\mathrm{M}$ \\
\hline 1 & 4 & 5 & 4 & 4 & 6 & 6 & 2 & 1.5 \\
\hline 2 & 4 & 3 & 5 & 3 & 6 & 6 & 2 & 2.5 \\
\hline 3 & 4 & 5 & 4 & 4.5 & 7 & 7 & 1 & 3 \\
\hline 4 & 6 & 4 & 4 & 4 & 6 & 6 & 3 & 3 \\
\hline 5 & 4 & 5 & 4 & 5 & 6 & 6 & 1 & 3 \\
\hline 6 & 4 & 4 & 4 & 4 & 6 & 6 & 2 & 2 \\
\hline 7 & 4 & 5 & 4 & 5 & 7 & 7 & 2 & 2 \\
\hline 8 & 7 & 7 & 6 & 6 & 6 & 6 & 2 & 1 \\
\hline 9 & 4 & 5 & 4 & 5 & 6 & 6 & 2 & 2 \\
\hline
\end{tabular}

${ }^{\text {a }}$ E: establishment stage.

${ }^{\mathrm{b}} \mathrm{M}$ : mature stage.

\subsection{Visual preference for color}

The mode, median, range, and inter-quartile range of the visual preferences for the photographs have been displayed in Table 2. The photos are numbered consecutively from 1 to 7, and in each case, photo 1 represents the image with the lowest level of color while photo 7 has the highest level of color. The scenes with the lowest amount and diversity of color were not as highly preferred as scenes with higher amounts and diversity of color. However, the ratings for scenes in the midrange of color tended to be more poorly differentiated by respondents, and there is no clear linear relationship between color and preference. Data categories were collapsed (e.g. Babbie, 2001) to assist identification of relationships. Fig. 3 grouped together ratings of 1-3 as 'dislike', and ratings of 5-7 as 'like', with 4 as neutral. For the establishment phase (Fig. 3a), there is an evident trend of increasing preference with increasing amount and diversity of color, with a weaker but still evident trend in the mature stage (Fig. 3b).

Spearman's rank coefficient identified a positive correlation between color and visual preference in the establishment stag $(\mathrm{R}=0.275)$ and the maturity stage $(\mathrm{R}=0.271)$, both significant at the $99 \%$ confidence interval.

\subsection{Visual preference for apparent human intent}

The mode, median, range, and inter-quartile range of the visual preferences for the photographs have been displayed in Table 3 . The photos are numbered consecutively from 1 to 9, and in each case, photo 1 represents the image with the lowest level of apparent human intent while photo 9 has the highest level. There was no clear pattern 
between level of apparent human intent and visual preference at either the establishment or mature stages, so data categories were again collapsed (Babbie, 2001). Fig. 4a and b grouped together ratings of 1-3 as 'dislike', and ratings of 5-7 as 'like', with 4 as neutral. Although it is clear that a number of the individual treatments have influenced visual preference, there was no consistent visible pattern evident from the data. However, this might be illustrating a limitation in the experimental design. While it was fairly straightforward to have a gradient of color in photographs, it was not as clear how to create a gradient of apparent human intent. If we instead view the landscape treatments individually, rather than as a gradient, photo 8 (a rocky ridge) stands out clearly as the most highly preferred image. Photos $2 \pm 6$ were different clustering options, and were rated approximately equal. The clustering alternatives, as a group, were more highly preferred than the natural regeneration alternatives (photos 1, 7 and 9).
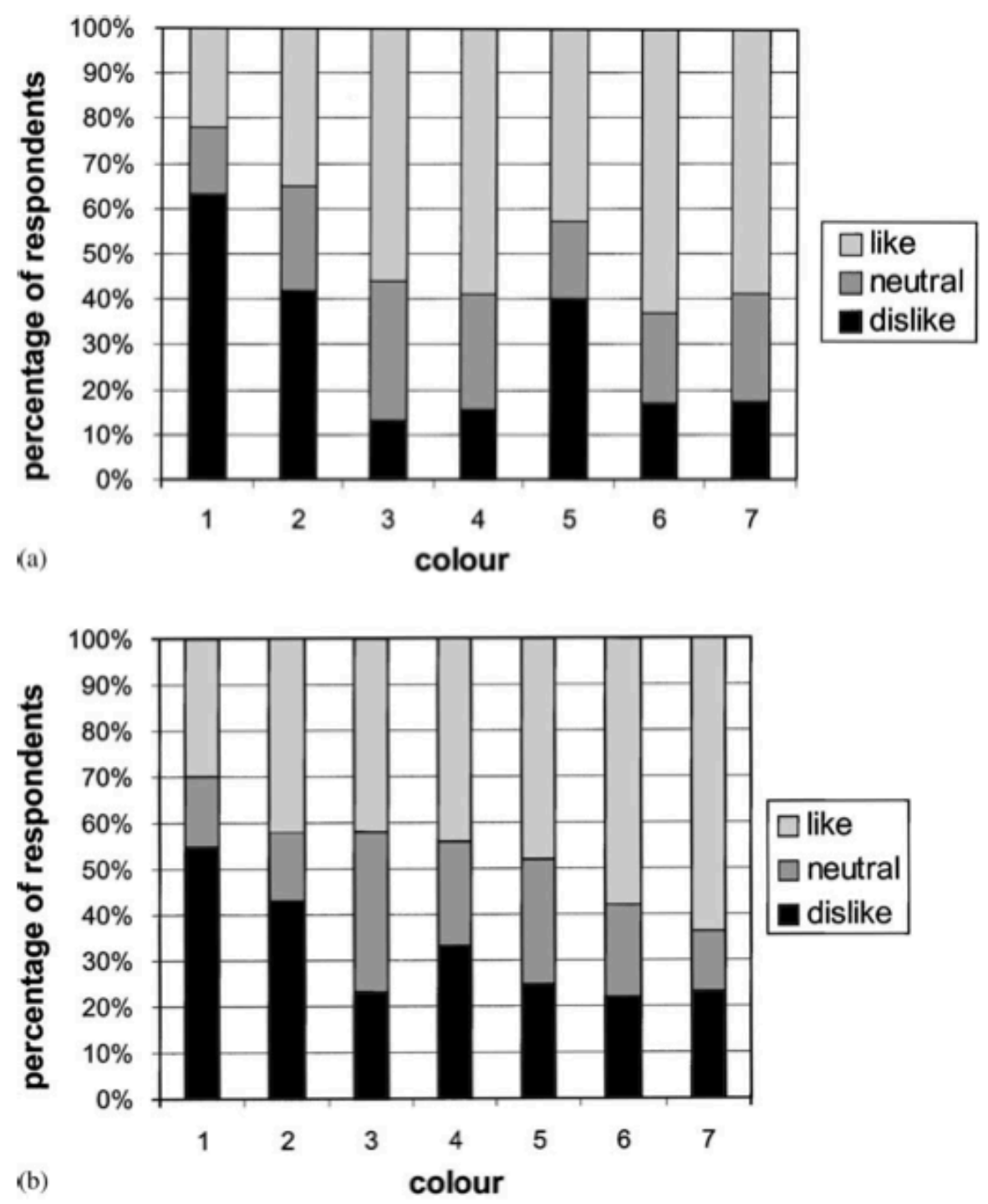

Fig. 3. (a) Percentages of responses grouped by like, neutral and dislike: establishment stage and (b) percentages of responses grouped by like, neutral and dislike: mature stage. 
Nontheless, the Spearman's rank coefficient analysis identified a positive correlation between apparent human intent and visual preference in the establishment $\operatorname{stag}(\mathrm{R}=0.119)$ and at the maturity stage $(\mathrm{R}=0.0262)$, both significant at the $99 \%$ confidence interval.

The lack of a clear pattern in the results led us to conduct a MDS analysis of the apparent human intent data. Images were analyzed according to similarities in their preference ratings and mapped in two dimensions (see Fig. 5a). The establishment phase yielded two main clusterings of photos. The first cluster in the upper right quadrant of the map consisted of all the regeneration alternatives. The second major cluster in the bottom left quadrant consisted of all the planted alternatives with the exception of the rocky ridge (photo 8) which is isolated in the upper left quadrant. We interpreted dimension 1 (yaxis) as "planted versus natural regeneration" and dimension 2 (x-axis) as either "level of human intervention" or 'level of apparent design". ‘Human intervention' is different from `human intent' in that it requires evidence of physical work or change.

In the mature stage, the photos were again clustered into two major groupings. Cluster 1 in the upper central portion of the map (Fig. 5b) contains the
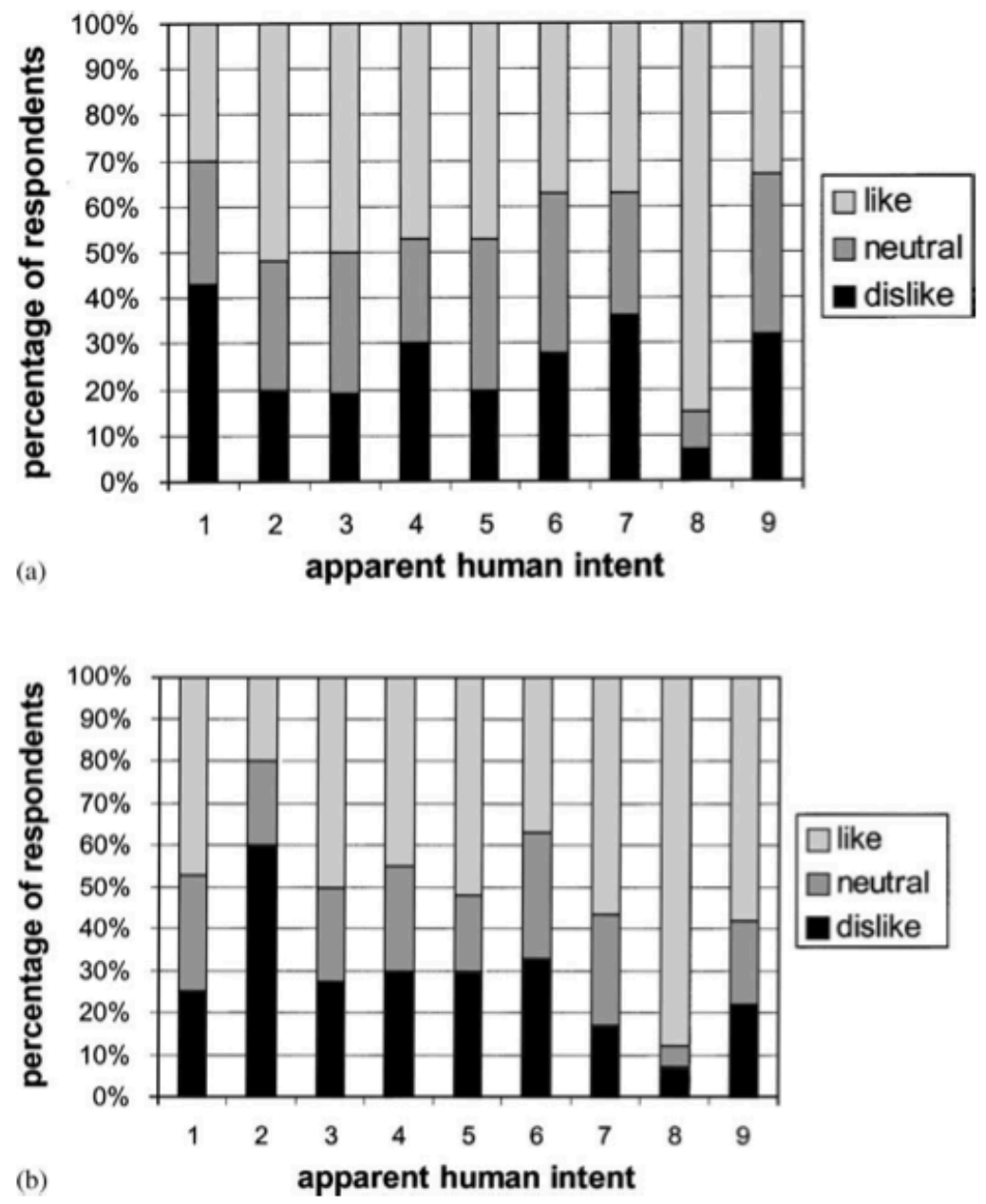

Fig. 4. (a) Percentages of responses grouped by like, neutral and dislike: establishment stage and (b) percentages of responses grouped by like, neutral and dislike: mature stage. 
majority of the planted alternatives, while cluster 2 in the lower central portion contains all the natural regeneration alternatives. We interpreted dimension 1 (y-axis) as "planted versus natural regeneration", as in the establishment phase. Along the horizontal dimension, images photos 2 and 8 are isolated at extreme ends of the scale, while clusters 1 and 2 are close together in the central area. Photo 2 represents the image of an untouched natural grassland with no apparent human intervention, while photo 8 represents the image of the rocky ridge. Thus, as in the establishment stage, dimension 2 (x-axis) appears to represent "level of human intervention" or "level of apparent design".

\subsection{Content analysis}

Four dominant themes were identified within respondents' comments concerning their reasons for selecting their most and least preferred photos: color, variety, naturalness/human care, and vegetation density. Most comments for photo sets 1 and 2 were related to color and variety, while in sets 3 and 4 there were more comments on degree of naturalness and human care.

Respondents commented on vegetation density approximately equally for all photo sets. They were more likely to comment negatively on sparseness in the early stages than they were to comment positively on fullness in the later stages. It seemed that if
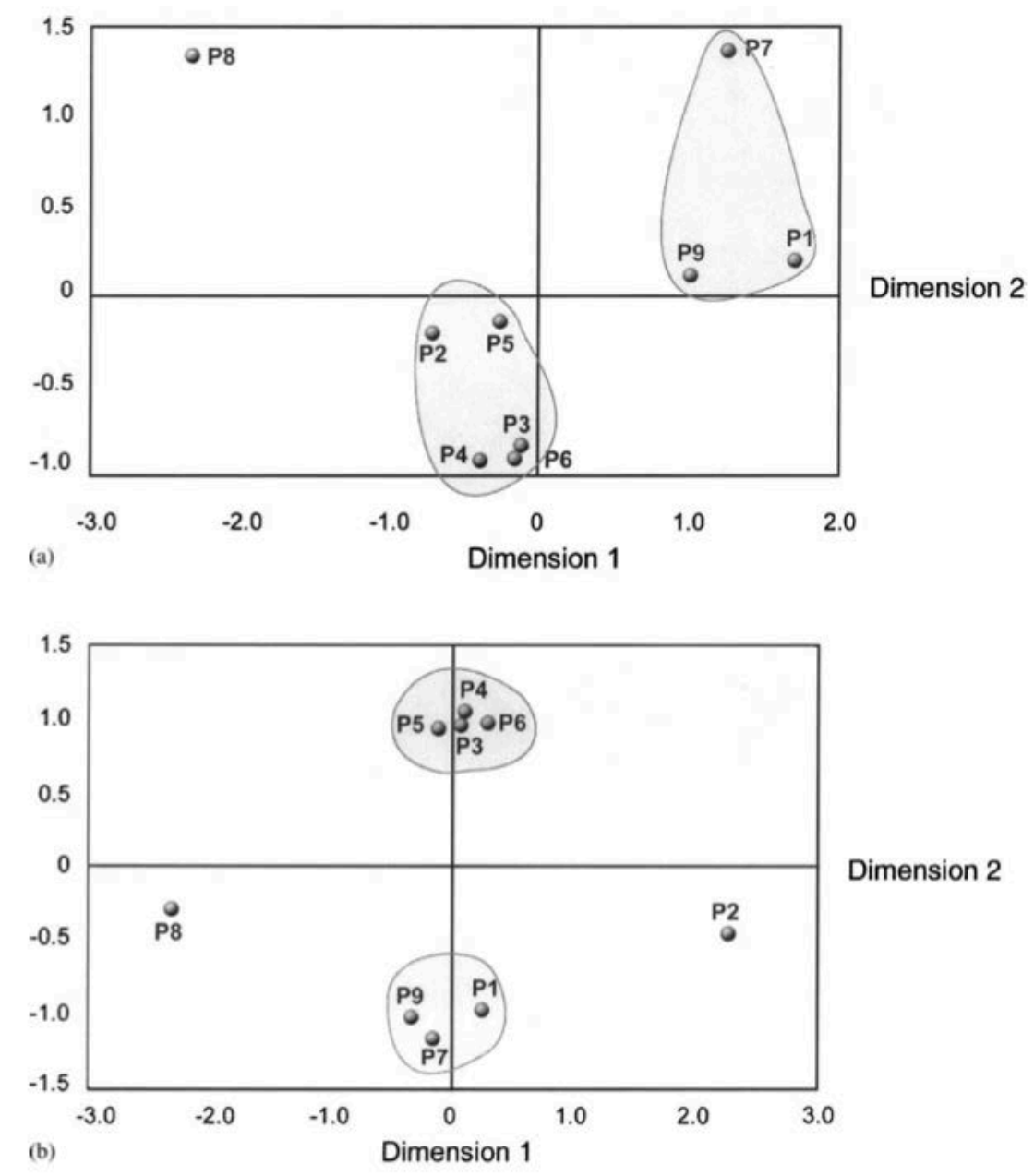

Fig. 5. MDS analysis: (a) establishment stage and (b) mature stage. 
vegetation density was considered sufficient, other factors became more noteworthy in regard to visual preference. Table 4 lists typical positive and negative comments within each theme, and Fig. $6 a$ and $b$ illustrates the relative percentage of descriptors for each photo set.

During the establishment phase, the single most common reason for liking or disliking a photo was "color". This supported the correlation analysis results. A considerable number $\square 18 \%$ ) commented negatively on the barrenness or sparseness of vegetation in the establishment stage. This suggests that rapid establishment and maintenance of ground cover is important for visual preference of rehabilitation projects.

Approximately an equal number of comments relating to color were made in the mature as in the establishment stage, however, noticeably fewer comments were made concerning variety in the mature stage. In fact, considerably more respondents $(20 \%$ versus $8 \%$ ) reported that they found some images to be too busy in the mature stage. Similarly, $11 \%$ of

Table 4

Content analysis of respondents' positive and negative comments

\begin{tabular}{|c|c|}
\hline Like & Dislike \\
\hline \multicolumn{2}{|l|}{ Theme 1: color } \\
\hline Lots of color, very colorful & Not enough color, not enough flowers \\
\hline Beautiful color, nice color & Too much green, monotone \\
\hline Pleasing color mixture & Too much color, too many flowers \\
\hline Lots of colorful wildflowers & Don't like color \\
\hline Yellow flowers & Too much of one color \\
\hline \multicolumn{2}{|l|}{ Theme 2: variety } \\
\hline Good variety, lots of variety & Not enough variety \\
\hline Nice mix, good assortment, diversified & Poorly contrasting \\
\hline Good contrast, balanced & Boring, drab, plain, dull, bland \\
\hline Different types of (textures, wildflowers, heights) & Too much variety \\
\hline Relief (topographic change) & Too many plant types \\
\hline Simple, not too busy/crowded, not over done & Too distracting, too overpowering, over done, too busy, too crowded \\
\hline \multicolumn{2}{|l|}{ Theme 3: naturalness/human care } \\
\hline Very natural, natural look & Unnatural looking, not natural \\
\hline Wildlife habitat, attract birds/butterflies & Looks like a flower/vegetable garden, linear rows of plants, too organised \\
\hline Rock garden effect, natural looking rocks, landscaping rocks & Too much like existing, looks like nothing was done, too industrial looking \\
\hline Well organised, easy to maintain, not overgrown & Overgrown, messy, field of weeds, looks too natural, scruffy, long grass \\
\hline Sign, bird box & Sign, bird box \\
\hline \multicolumn{2}{|l|}{ Theme 4: vegetation density } \\
\hline Lush & Too sparse, bare, empty, barren \\
\hline Luscious vegetation & Too many open spaces \\
\hline Coverage full & Too wide spacing \\
\hline Looks better when full & Too much exposed ground \\
\hline Looks better when full & \\
\hline
\end{tabular}

respondents (compared with $2 \%$ in the establishment stage) indentified "simple" as a positive characteristic of images that they preferred in the mature stage. These results are 
consistent with earlier findings (e.g. Kaplan and Kaplan, 1989) that people tend to prefer images in the mid-range of complexity, and indicate that for mature grassland communities a threshold exists beyond which additional color and/ or variety are not preferred.

The natural appearance of the landscape seems to be more important in the mature stages, with four times as many respondents identifying it as a positive characteristic.

\section{Discussion}

Content analysis results indicated that rehabilitating landscapes were perceived as messy in the context of an operational industrial facility, and that their messy appearance was considered problematic. 'Messy appearance' was the most commonly stated problem (25\%) that employees foresaw with rehabilitation. However, when describing what they disliked about their least preferred study image, they only identified messy appearance 49\% of the time. Employees seemed to anticipate that the landscape will look messier than the simulations indicated that it would be.

The effect of apparent human intent on visual preference was mixed. Some indicators, such as the addition of the large rock formation, had substantial positive effects on visual preference. Similarly, the addition of color had significant positive effects. Clustering, however, had little discernible effect at all. Reactions to the addition of the bird box and interpretive signage were generally positive, but varied widely.
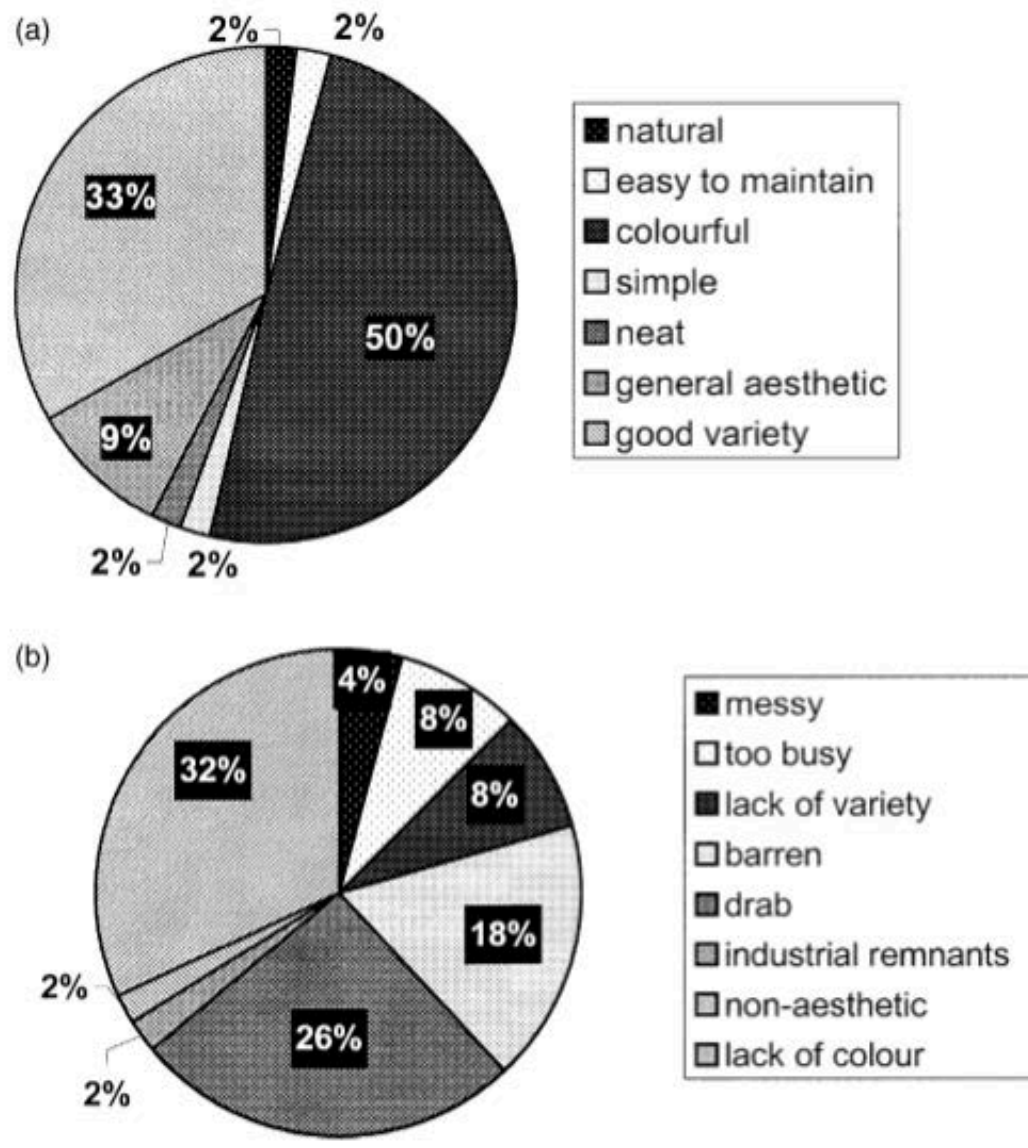

Fig. 6. (a) Positive and (b) negative descriptors of photo set 1. 


\section{Implications for design}

This research has demonstrated that vernacular cues to care such as the addition of color in the form of bold flowering forbs, and cultural elements such as bird boxes and rocks can significantly improve visual preference in this context. Many of these interventions will not significantly reduce the ecological functioning of the landscape, and can most likely be confined to high visibility areas.

Content analysis results indicated that sparseness of vegetation was a major reason for negative preferences in the establishment stage. Thus, any techniques that contribute to the rapid formation of ground cover should considerably improve visual preferences for rehabilitating landscapes during the establishment period. Such techniques could include the use of a rapidly establishing nurse crop, or a number of no-till methods which would preserve the existing vegetative ground cover.

Especially in the establishment stage, increasing color was quite clearly linked to increasing visual preference. This finding indicates that any methods than maximize color in the early stages would be beneficial.

The considerably stronger effect on visual preference resulting from the addition of the rocky ridge in comparison with all other elements in this study might suggest that some large-scale elements are desirable in large open landscapes in order to add interest and diversity to the site.

Natural regeneration solutions were particularly not preferred in the establishment stage. Respondents seemed to desire a more active approach to the rehabilitation of their

site. Although the scale of most industrial rehabilitation projects necessitates the reliance on natural regeneration processes in order to maintain budgets, a few high profile plantings in highly visible areas during the early stages of a project might be very important in maintaining the impressions that 'something is being done'.

\section{Limitations of the study}

The findings of this study are based on a specific subject group (Cytec employees) and caution should be exercised in generalizing results to other subject groups. The study was also limited to the degree that photo surrogates used are representative both of the site experience of the scene and the rehabilitation scenario simulated. A wider variety of scenes and viewpoints would improve representativeness, as well as the ability to generalize results.

Differences between study photographs were often subtle, and in some cases it may have been difficult for subjects to differentiate among them. This assertion is supported by respondents' comments and the high number of tied ratings. Given the attention necessary to differentiate the photographs, respondent fatigue may have been a factor in some cases. 


\section{Future research}

The application of a similar methodology to examine further design variables would be very valuable. Some variables might prove to be more significant in predicting preference than others, and additional research will be required to determine the relative importance of the different variables. Similarly, this methodology should be applied to a wider range of landscape viewpoints to allow for greater analysis at the landscape level.

\section{Summary and conclusions}

It was apparent from this research that there exists a wide range of visual preferences for rehabilitated industrial landscapes, as well as a range of factors affecting these preferences. There was substantial disagreement across respondents in this study as evidenced by the large ranges in preference ratings, as well as the sometimes contradictory comments made in answers to survey questions regarding likes and dislikes.

It was also apparent that Cytec employees did not find ecological rehabilitation landscapes necessarily beautiful. They seemed to prefer an enhanced nature that was more colorful, more deliberate, and less messy than the natural indigenous community. Cytec employees expressed a desire for their site to look natural, but not too natural. Further, many of the desires were potentially conflicting, such as the desire to promote wildlife and the desire for neatness.

It appeared that an important criterion in evaluating rehabilitating industrial landscapes is whether they are being actively rehabilitated by human intervention, or allowed to naturally regenerate. Although strong indicators of human intent such as interpretive signage do improve visual preference for naturally regenerating landscapes, these are still not as preferred as landscapes which show some indications of more active intervention in the establishment stages.

Results indicate that the role of apparent human intent in visual preference for rehabilitating industrial landscapes is very complex, and that there is a place for thoughtful design which considers aesthetic expectations, as well as perceived appropriate levels of human intervention. It might be necessary to make some concessions to ecological integrity and diversity in highly visible areas in order to meet aesthetic expectations. Incorporation of the vernacular cues to care explored in this study provides a good starting point for improving the visual preferences for rehabilitating industrial landscapes. 


\section{References}

Babbie, E., 2001. The Practice of Social Research, 9th Edition. Wadsworth/Thompson Learning, Belmont, USA.

Barro, S.C., Bright, A.D., 1998. Public views on ecological restoration: a snapshot from the Chicago arsea. Restorat. Manage. Notes $16 \square 1$ ), 59-65.

Gallager, T.J., 1977. Visual preference for alternative natural landscapes. Ph.D.

Dissertation. University of Michigan, Ann Arbor, MI, USA.

Gobster, P.H., 1994. The urban savanna: reuniting ecological preference and function. Restorat. Manage. Notes $12 \square 1$ ), 64-71.

Hough, M., 1989. City Form and Natural Process. Chapman \& Hall, New York.

Kaplan, R., Kaplan, S., 1989. Experience of Nature. Cambridge University Press, New York.

Kaplan, R., 1993. The role of nature in the context of the workplace. Landscape Urban Plan. 26, 193-201.70 D.E. Hands, R.D. Brown / Landscape and Urban Planning 58 $\square$ 2002) $57-70$

Keyes, B.E., 1984. Visual preferences of a forest trail environment. Master's Thesis, University of Tennessee, Knoxville, TN, USA, unpublished .

Maag, S.L., 1995. Perceptions of prairie in corporate settings: a study in adaptation. Masters Thesis, University of Minnesota, MN, USA, unpublished.

Nassauer, J.I., 1988. The aesthetics of horticulture: neatness as a form of care. J. Environ. Manage. 17, 1-16.

Nassauer, J.I., 1995. Messy ecosystems, orderly frames. Landscape J. $14 \square 2$ ), 161-170.

Nassauer, J.I., 1997. Placing Nature: Culture and Landscape Ecology. Island Press, Washington, DC, USA.

Orland, B., 1994. Synthetic landscapes: a review of video-imaging applications in environmental perception research, planning and design. In: Daniel, T., Orland, B. Eds.), Design Support 2001: Workshop Notes, Data Visualization, pp. 213-248.

Raffetto, J., 1993. Perceptions of ecological restorations in urban parks. In: Gobster P.H. Ed.), Managing Urban and High-Use Recreation Settings: Selected Papers from the Urban Forestry and Ethnic Minorities and the Environment Paper Sessions. USDA Forest Service, North Central Forest Experiment Station, Chicago. 
Schulhof, R., 1989. Public perceptions of native vegetation. Restorat. Manage. Notes 7 $\square 2), 69-72$.

Ye, W., 1993. Relationship of image quality and viewer's perceived realism of images derived from computer-imaging technology. Master's Thesis, University of Guelph, Guelph, ON, USA, unpublished. 